\title{
CHARACTERISATION OF VERY STRONGLY PERFECT GRAPHS
}

\author{
G.R. Ganesh Gandal \\ Department of Mathematics, \\ Sathyabama Institute of Science and Technology, Chennai, India.

\section{R. Mary Jeya Jothi} \\ Department of Mathematics, \\ Sathyabama Institute of Science and Technology, Chennai, India.
}

\begin{abstract}
A graph $G$ is said to be strongly perfect if every induced sub graph $H$ has an independent set, meeting all maximal complete sub graphs of $H$. A strongly perfect graph is said to be very strongly perfect if it contains good independent set. It follows that every very strongly perfect graph is perfect not conversely. For example, the compliment of even cycle of length more than 4 is not very strongly perfect though it is perfect. In this paper, we characterise the classes of very strongly perfect graphs like cyclic graph, tadpole graph, barbell graph and friendship graph along with its independence number.
\end{abstract}

\section{AMS Classification: 05C17.}

Keywords: very strongly perfect graph, cyclic graph, complete graph.

Cite this Article: G.R. Ganesh Gandal, R. Mary Jeya Jothi, Characterisation of Very Strongly Perfect Graphs. International Journal of Mechanical Engineering and Technology 11(1), 2020, pp. 40-46.

https://iaeme.com/Home/issue/IJMET?Volume=11\&Issue=1

\section{INTRODUCTION}

In this paper, graphs are finite and simple. A path in a graph $\mathrm{G}(\mathrm{V}, \mathrm{E})$ is an alternating sequence of vertices and edges. Every path contains maximal clique of size two i.e. $\mathrm{k}_{2}$ such that all alternating set of vertices form an independent set which meets all maximal cliques. Therefore every path is very strongly perfect. A simple graph $\mathrm{G}$ is said to be complete if every distinct pair of vertices are adjacent. The complete graph on n-vertices is denoted by $K_{n}$. Since in $K_{n}$, every vertex is an independent set which meets its maximal clique (complete graph itself), every complete graph is very strongly perfect. A clique in a graph $G$ is a maximal complete sub graph. An independent set is a set of vertices such that no two of which are adjacent. An independent set is said to be good independent if each of its vertex meets 
maximal clique. The number of vertices in a largest independent set of graph $\mathrm{G}$ is called independence number denoted by $\psi(G)$.

The theory of perfect graph deals with the theoretical concepts of a clique and independent set. An application of perfect graph is in an urban science problem involving optimal routing of garbage trucks. Also perfect graphs can be used to solve polynomial time algorithm, based on the ellipsoid method, for finding a maximum stable set and minimal colouring. The major role of graph theory in computer applications is to develop the graph algorithms like data mining, image segmentation, networking, clustering, image capturing etc.

Due to the applications of perfect graphs, it is necessary to investigate its subclasses too. As very strongly perfect graph is the subclass of perfect graphs, here it is investigated the same graph.

\section{VERY STRONGLY PERFECT GRAPH (VSP)}

A graph $\mathrm{G}$ is said to be very strongly perfect if in every induced sub graph $\mathrm{H}$, every vertex of $\mathrm{H}$ belongs to an independent set of $\mathrm{H}$ meeting all maximal cliques of $\mathrm{H}$. Fig. 1 and 2 illustrate both very strongly and non very strongly perfect graphs.

\section{Example 1}

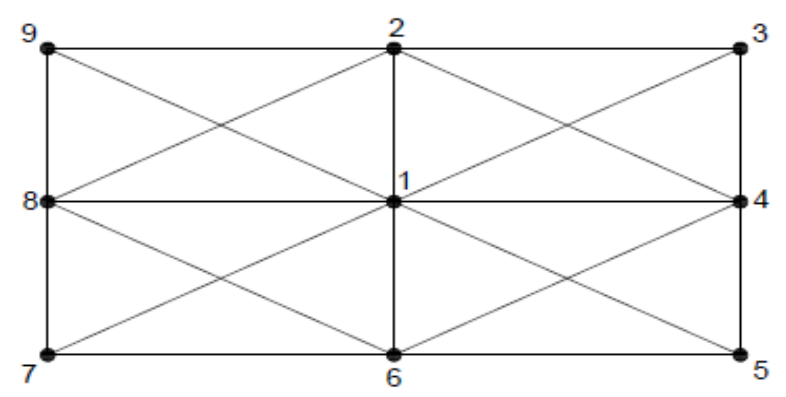

Figure 1: Very Strongly Perfect Graph

Here, $\{1\}$ is a independent set which meets all the maximal cliques of graph $\mathrm{G}$.

\section{Example 2}

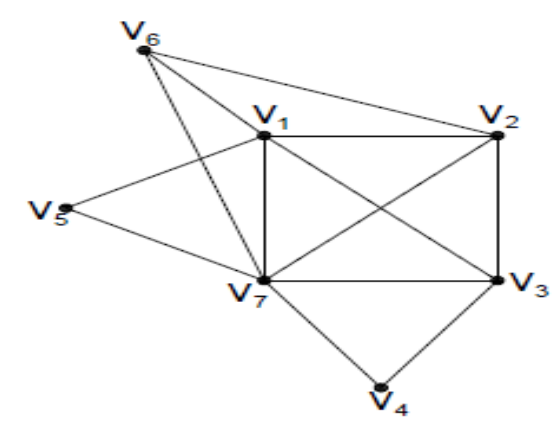

Figure 2: Non-Very Strongly Perfect Graph

Here, $\left\{\mathrm{v}_{4}, \mathrm{v}_{5}, \mathrm{v}_{6}\right\}$ is an independent set which does not meet all maximal cliques. 


\section{CYCLE}

A Cycle graph or Circular graph is a graph that consists of a single cycle, or in other words, some number of vertices connected in a closed chain (A path in which terminal vertices are coinciding is called cycle). The cycle graph with $n$ vertices is denoted by $C_{n}$. The even cycle is a cycle with even number of vertices and the odd cycle is a cycle with odd number of vertices. The number of vertices in a $C_{n}$ equals the number of edges, and every vertex has degree 2; that is, every vertex has exactly two edges incident with it. The following figure illustrates the cycle graph $\mathrm{C}_{6}$.

\section{Example 3}

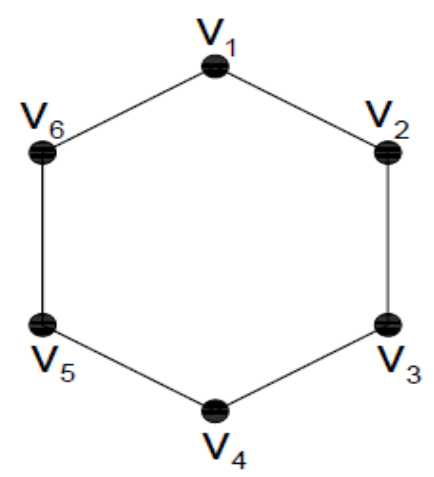

Figure 3: $\mathrm{C}_{6}$

\subsection{Theorem}

Every Even Cycle is very strongly perfect.

\section{Proof:}

Consider the cycle $\mathrm{C}_{\mathrm{n}}$ of even length.

Therefore, $C_{n}=\left\{v_{1}, v_{2}, v_{3} \ldots \ldots v_{n}, v_{1}\right\}$, where $n$ is even.

Let $\mathrm{I}=$ independent set (set of nonadjacent vertices).

Without loss of generality, let $v_{1} \in I, v_{2} \notin I$ and so on.

Hence for all $j \in(1,2,3, . ., n)$

We get, $v_{j} \in I$ If $\mathrm{j}$ is odd and $v_{j} \notin I$ if $\mathrm{j}$ is even.

As $\mathrm{n}$ is even, $v_{n} \notin I$.

Therefore $I=\left\{v_{j} / j\right.$ is odd $\}$ which meets all the maximal cliques of length two (i.e.) $k_{2}$. Hence, $\mathrm{G}$ is very strongly perfect.

\subsection{Theorem}

Every Odd Cycle is non-very strongly perfect.

Proof:

Consider the cycle $\mathrm{C}_{\mathrm{n}}$ of odd length.

Therefore, $\mathrm{C}_{\mathrm{n}}=\left\{\mathrm{v}_{1}, \mathrm{v}_{2}, \mathrm{v}_{3} \ldots \ldots \mathrm{v}_{\mathrm{n}}, \mathrm{v}_{1}\right\}$, where $\mathrm{n}$ is odd.

Let I be the independent set such that $v_{1} \in I, v_{2} \notin I$ and so on. 
Hence for all $k \in(1,2,3, . ., n)$, we get $v_{k} \in I$ if $\mathrm{k}$ is odd and $v_{k} \notin I$ if $\mathrm{k}$ is even.

As $\mathrm{n}$ is odd, $v_{n} \in I$.

Therefore there exists an edge $\mathrm{e}=\mathrm{v}_{\mathrm{n}} \mathrm{v}_{1}$ in $\mathrm{G}$ belongs to independent set $\mathrm{I}$.

That proves I contains two adjacent vertices.

Which gives contradiction to the fact that $\mathrm{I}$ is an independent set.

Hence, $G$ is not very strongly perfect.

\subsection{Proposition}

Every even cycle graph $\mathrm{C}_{\mathrm{n}}$ has the cardinality of independence number $\frac{n}{2}$.

\section{Proof:}

Let $C_{n}$ be the cycle containing $n$ number of vertices, where $n$ is even.

Let $\mathrm{I}$ be the independent set containing alternating vertices of $\mathrm{C}_{\mathrm{n}}$ which meets all cliques.

Since $\mathrm{G}$ has n vertices, I contains $\frac{n}{2}$ vertices which is maximum.

Hence the proof.

\section{TADPOLE GRAPH}

The $(\mathrm{m}, \mathrm{n})$ - Tadpole graph, also called a dragon graph, is the graph obtained by joining a cycle graph $\mathrm{C}_{\mathrm{m}}$ to a path graph $\mathrm{P}_{\mathrm{n}}$ with a bridge and it is denoted by $\mathrm{T}_{\mathrm{m}, \mathrm{n}}$. The $(\mathrm{m}, 1)$-tadpole graph is sometimes known as the m-pan graph. The particular cases of the $(3,1)$ - and $(4,1)$ tadpole graphs are also known as the paw graph and banner graph, respectively. The tadpole graph $\mathrm{T}_{4,4}$ is illustrated in fig. 4 .

\section{Example 4}

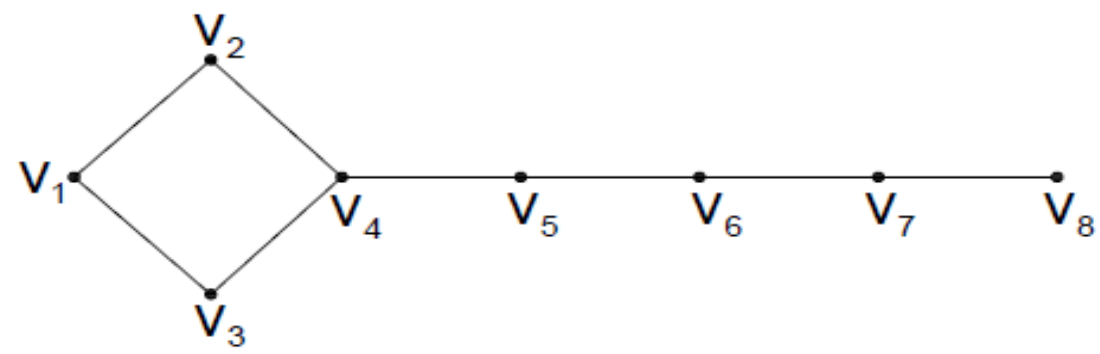

Figure 4: $\mathrm{T}_{4,4}$

Independent set $=\left\{\mathrm{v}_{1}, \mathrm{v}_{4}, \mathrm{v}_{6}, \mathrm{v}_{8}\right\}$ meets all maximal cliques.

\subsection{Theorem}

Every tadpole graph $\mathrm{T}_{2 \mathrm{~m}, \mathrm{n}}$ is very strongly perfect.

Proof:

Let $\mathrm{G}$ be a tadpole graph.

Since $\mathrm{G}$ is constructed by joining a cycle of length $2 \mathrm{~m}$ i.e. $\mathrm{C}_{2 \mathrm{~m}}$ with a path of length $\mathrm{n}$ (i.e.,) $P_{n}$. 
Also, we see in theorem 3.1 that every even cycle is very strongly perfect.

As $P_{n}$ is a path of $n$ vertices, therefore every alternating set of vertices from the path forms the independent set I which meets maximal clique $\mathrm{k}_{2}$.

Since $\mathrm{G}$ is the union of cycle $\mathrm{C}_{2 \mathrm{~m}}$ and path $\mathrm{P}_{\mathrm{n}}$, both are very strongly perfect.

Hence, $\mathrm{G}$ is a very strongly perfect.

\subsection{Theorem}

Every tadpole graph $\mathrm{T}_{2 \mathrm{~m}+1, \mathrm{n}}$ is non-very strongly perfect.

\section{Proof:}

Let $\mathrm{G}$ be a tadpole graph.

$\mathrm{G}$ is constructed by joining a cycle of length $2 m+1$ i.e. $\mathrm{C}_{2 m+1}$ with a path of length $n$ i.e. $\mathrm{P}_{\mathrm{n}} \cdot \mathrm{C}_{2 \mathrm{~m}+1}$ is a odd cycle which is a non- very strongly perfect graph and every path is very strongly perfect graph .The union of $\mathrm{C}_{2 \mathrm{~m}+1}$ and $\mathrm{P}_{\mathrm{n}}$ results a non-very strongly perfect graph.

Hence, $\mathrm{G}$ is non- very strongly perfect graph.

\subsection{Proposition}

Independence number of tadpole graph $\mathrm{T}_{2 \mathrm{~m}, \mathrm{n}}$ is $m+\frac{n}{2}$.

\section{Proof:}

Let $\mathrm{T}_{2 \mathrm{~m}, \mathrm{n}}$ be a tadpole graph.

$\Rightarrow$ there exists a cycle of length $2 \mathrm{~m}$ which gives m number of vertices and there is a path of length $\mathrm{n}$ which gives $\frac{n}{2}$ number of vertices belonging to an independent set. Therefore maximum number of vertices belonging to an independent set is $m+\frac{n}{2}$.

Hence the proof.

\section{BARBELL GRAPH}

The n-barbell graph is the simple graph obtained by connecting two copies of a complete graph $\mathrm{K}_{\mathrm{n}}$ by a bridge. Bridge is an edge whose deletion disconnects the graph. Fig. 5 illustrates the 4-barball graph.

\section{Example 5}

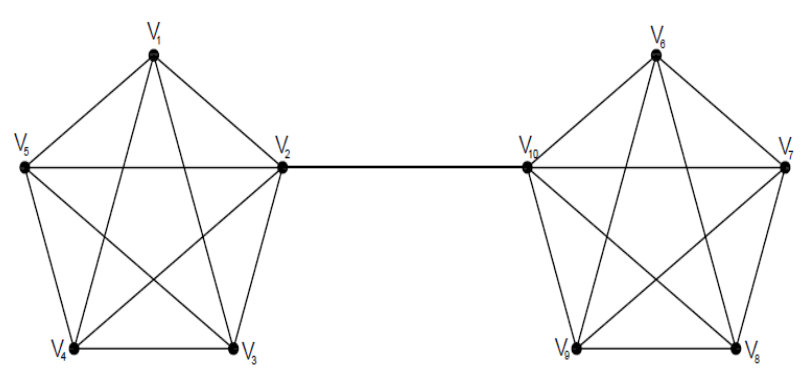

Figure 5: 4 - Barbell Graph 


\subsection{Theorem}

Every n-barbell graph is very strongly perfect.

Proof:

Let $\mathrm{G}$ be an $\mathrm{n}$-barbell graph.

Every complete graph is very strongly perfect.

If two very strongly perfect graphs are joined by a bridge (i.e.) $\mathrm{K}_{2}$, the resultant graph becomes very strongly perfect graph.

Hence, $G$ is very strongly perfect.

\subsection{Proposition}

Every barbell graph has independence number two.

\section{Proof:}

Every barbell graph is obtained by connecting two copies of complete graph $\mathrm{K}_{\mathrm{n}}$ by a bridge. As each complete graph gives only one vertex which belongs to independent set, therefore there exists a largest independent set of size two.

Hence the proof.

\section{FRIENDSHIP GRAPH}

A Friendship graph $\mathrm{F}_{\mathrm{k}}$ of $2 \mathrm{k}+1$ vertices is the graph obtained by taking k-copies of the cycle graph $\mathrm{C}_{3}$, with a vertex in common, (i.e.) the graph of $\mathrm{k}$-triangles intersecting in a single vertex. Hence every friendship graph contains a single vertex which is adjacent to all the remaining vertices with unit distance. It is a graph with girth 3 and diameter 2.Friendship graph $\mathrm{F}_{6}$ is illustrated in fig. 6 .

\section{Example 6}

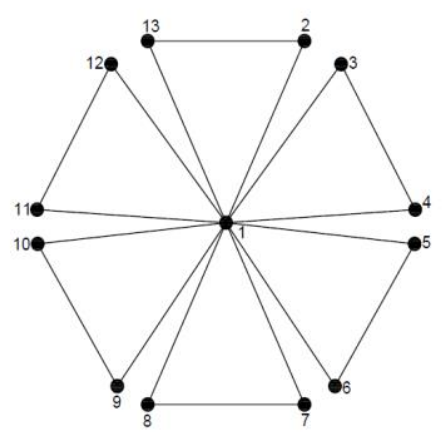

Figure 6: $\mathrm{F}_{6}$

\subsection{Theorem}

Every Friendship Graph is very strongly perfect.

Proof:

Let $\mathrm{G}$ be a friendship graph

$\Rightarrow$ there exists a vertex $\mathrm{v} \in \mathrm{V}$, which is adjacent to all the other vertices of $\mathrm{G}$.

$\Rightarrow\{v\}$ is an independent set of cardinality one which meets all the maximal cliques of size three.

$\Rightarrow \mathrm{G}$ is very strongly perfect. 


\subsection{Proposition}

Every friendship graph $\mathrm{F}_{\mathrm{k}}$ has independence number $\mathrm{k}$.

Proof:

Let $\mathrm{G}$ be a friendship graph $\mathrm{F}_{\mathrm{k}}$.

$\Rightarrow \mathrm{G}$ has $\mathrm{k}$ copies of maximal cliques of size three (i.e $\mathrm{k}_{3}$ ).

$\Rightarrow$ in each $\mathrm{k}_{3}$, there is only one vertex which lies in independent set.

Therefore independent set consists of at most $\mathrm{k}$ number of vertices.

Hence the proof.

\section{CONCLUSION}

Here, it is discussed the structural properties of very strongly perfect graphs on cycle, tadpole, barbell and friendship graphs. In future, this investigation will be more applicable for the remaining graph classes also.

\section{REFERENCES}

[1] G. Ravindra, Meyniel graphs are strongly Perfect, Journal of Combinatorial Theory, Series 33, 187-190(1982).

[2] Stefan Hougardy, "Classes of perfect graphs." Discrete Mathematics 306 (20) 062529 2571.

[3] R. Mary Jeya Jothi and A. Amutha, An investigation on the characterization of super strongly perfect graphs on trees, Proceedings of the Second International Conference on Soft Computing for Problem Solving (Soc ProS 2012), Advances in Intelligent Systems and Computing, 236 (2012), 279 - 285, Springer Publications.

[4] R. Mary Jeya Jothi, A. Amutha, SSP Structure of Some Graph Classes, International Journal of pure and Applied Mathematics,101(2015), 939-948.

[5] C. Berge and P. Duchet, Strongly Perfect Graphs, Annals of discrete mathematics 21(1984), 57-61. 